\title{
Electron-Polarization Coupling in Superconductor-Ferroelectric Superlattices
}

\author{
N. Pavlenko* and F. Schwabl \\ Institut für Theoretische Physik T34, Physik-Department der TU München, \\ James-Franck-Strasse, D-85747 Garching b. München, Germany
}

(Dated: November 8, 2018)

\begin{abstract}
We present a phenomenological model of periodic ferroelectric-superconductor (FE-S) heterostructures containing two alternating ferroelectric and superconducting layers. The interaction at the FE-S contacts is described as a coupling of the local carrier density of the superconductor with the spontaneous ferroelectric polarization near the FE-S interface. We obtain a stable symmetric domain-type phase exhibiting a contact-induced polarization and the ferroelectric domain structure at temperatures above the bulk ferroelectric transition temperature. With increasing coupling energy, we find the appearance of the ferroelectric phase coexisting with the suppressed superconductivity in the S-film. The system is analyzed for different thicknesses of the FE- and S-films demonstrating the dramatic change of the topology of the phase diagrams with a variation of the layers thickness. The results are expected to shed light on processes occurring in high-temperature superconducting films grown on perovskite alloy-substrates exhibiting ferroelectric properties at lower temperatures.

PACS numbers: 74.81.-g,74.78.Fk,77.80.-e
\end{abstract}

\section{INTRODUCTION}

Epitaxial combination of superconducting films and ferroelectric layers is promising for construction of effective microwave devices. Especially advantageous is the application of small-loss high-temperature cuprate superconductors $(\mathrm{YBCO})^{1.2}$ and perovskite ferroelectric alloys like $\mathrm{Ba}_{x} \mathrm{Sr}_{1-x} \mathrm{TiO}_{3}$ (BST) and $\mathrm{Pb}\left(\mathrm{Zr}_{x} \mathrm{Ti}_{1-x}\right) \mathrm{O}_{3}$ (PZT), in heterogeneous miltilayers. As both of these compounds have similar perovskite-type crystal structure with small lattice mismatch, the well oriented BST and PZT thin layers can be grown on the surfaces of $\mathrm{YBCO}^{3.4 .5 .6}$. In addition, the role of strains and off-stoichiometry structural defects at the BST(PZT)/YBCO-interfaces can be minimized by the use of growing methods such as laser ablation for fabrication of the miltilayered structures ${ }^{3}$.

Since the 1960s, the strong interest in developing electric field-effect devices based on ferroelectrics has been stimulated by observations of shifts of superconducting transition temperature under a switch of the spontaneous polarization. Superconducting field-effect transistors (SUFETs) cannot be realized with conventional superconductors since the large coherence lengths and high carrier density of the latter result in negligibly small effect of the polarization field ${ }^{7.8}$. In contrast, the carrier density and coherence lengths of high- $\mathrm{T}_{c}$ cuprates (HTS) which are two orders of magnitude lower than those in conventional superconductors ${ }^{9}$, , have made their use in SUFETs very attractive ${ }^{10}$.

For example, it was demonstrated in Ref. 11 1213 , that the critical temperature of YBCO films could be shifted by about $6 \mathrm{~K}$ through the polarization in the $\mathrm{BaTiO}_{3}$ substrate. As was assumed in ${ }^{12,14,15,16}$, the increase of $T_{c}^{S}$ is connected with the formation of the accumulation layers (with charge carrier density enhanced due to the polarization directed toward the interface), whereas the polarization in the opposite direction induces a depletion layer with higher resistance and lower $T_{c}^{S}$. Besides critical temperature, ferroelectric polarization affects transport properties of SUFETs leading to a change in resistance of $9-25 \%$ for PZT-YBCO and PZT-NdBa $\mathrm{Cu}_{3} \mathrm{O}_{7-\delta}(\mathrm{NBCO})$ heterostructures ${ }^{17.18}$ and showing polarization-induced memory effects in resistance and current, which suggests their possible storage applications 19 .

In the case when the polarization is parallel to the FE-S interface, the use of the zero- $P$ boundary conditions $(\boldsymbol{P}=0$ at the electrode) results in a weak effect of the FE-layers scarcely affecting the behavior of the Sfilms. On the other hand, the influence of the FE-layer should be essentially strong when the FE-polarization vector $\boldsymbol{P}$ is perpendicular to the FE-S interface. Due to the specific perovskite-like structure, the properties of the high- $\mathrm{T}_{c}$ materials are believed to be very close to the perovskite-type ferroelectrics. This fact stimulated discussions about a coexistence of ferroelectricity and superconductivity and a possible ferroelectricity of HTScompounds 20.21 .22 . As a consequence, the ferroelectric polarization has been suggested to be nonzero at the interface with the HTS-layer with even the possibility of penetration inside the superconductor ${ }^{23}$.

Recent studies of ferroelectric-HTS layered structures were focused on investigations of the role of free $P$ boundary conditions (non-zero $P$ ) on dielectric properties of FE-layers ${ }^{1,2,23}$. However, little attention has been paid to the behavior of superconducting films as well as to the effect of the FE-S interface on ferroelectric structural transformations.

In this work, we develop a phenomenological model based on the Ginzburg-Landau theory, for the FE-layers sandwiched between superconducting films. As compared to the microscopic approach proposed in Ref. 24 where the S-layer was described as two S-planes with a 
possibility of a charge transfer between the planes and the interior of the layer, the proposed model allows one to study directly the behavior of the system for different thicknesses of the S-layer in the vicinity of the ferroelectric transition temperature in the FE-film, which is assumed to be close to the transition temperature of the superconductor. We note that for HTS-compounds the condition $\xi_{S}(T) \gg \xi_{S}^{0}$ for the Ginzburg-Landau theory is valid over a much wider temperature range than in conventional superconductors ${ }^{29}$. We focus primarily on the case when the penetration depth of the polarizationinduced electric field is larger than the superconducting coherence length $\left(l_{T F} / \xi_{S}^{0} \gg 1\right)$, which is also typically realized in HTS layers.

The paper is organized as follows. In section III we introduce the Ginzburg-Landau energy functional for the FE-S heterostructure. We derive the Ginzburg-Landau equations and elucidate the nature of the boundary conditions. In section[II] we analyze in details the results for the case when the bulk ferroelectric transition temperature lies below the temperature of the superconducting transition. We discuss possible phases which can be stabilized in the system depending on the values of the temperature and the coupling energy. Corresponding phase diagrams are analyzed in section $\amalg$ for different thicknesses of the FE- and S-layers. We discuss here also the thicknesses-dependences of the ferroelectric transition temperature. Concluding remarks are presented in section ??.

\section{THE MODEL}

We consider a system containing periodically alternating ferroelectric (FE) layer sandwiched between superconducting (S) films (shown in Fig. (1) infinitely extended in the $x$ and $y$ directions. We assume here that the polarization $\boldsymbol{P}=(0,0, P(z))$ is directed perpendicular to the FE-S interface. The thicknesses of the FE- and Slayers are given by $L_{1}$ and $L_{2}$ respectively, and thus the total width of the supercell is: $L=L_{1}+L_{2}$. Generally, the whole sandwich structure can be described by the following Ginzburg-Landau functional

$$
F=F_{F E}+F_{S}+F_{\text {int }} .
$$

The ferroelectric part of the free energy (11) is given by

$F_{F E}=\int_{0}^{L_{1}} d z\left[\frac{1}{2} a_{F} P^{2}(z)+\frac{1}{4} b_{F} P^{4}(z)+\frac{1}{2} c_{F}\left(\frac{d P(z)}{d z}\right)^{2}\right]$,

where $a_{F}=a_{F}^{0}\left(T-T_{c}^{F}\right)$ is the inverse of the ferroelectric susceptibility and $T_{c}^{F}$ denotes the bulk Curie temperature. For simplicity, we do not include the sixth-order term into the $P$-expansion (2), restricting our analysis to second-order phase transformations. Note that since the polarization depends on $z$ only, the last term in (2) simplifies to $\nabla \boldsymbol{P}=\frac{d P(z)}{d z}$.

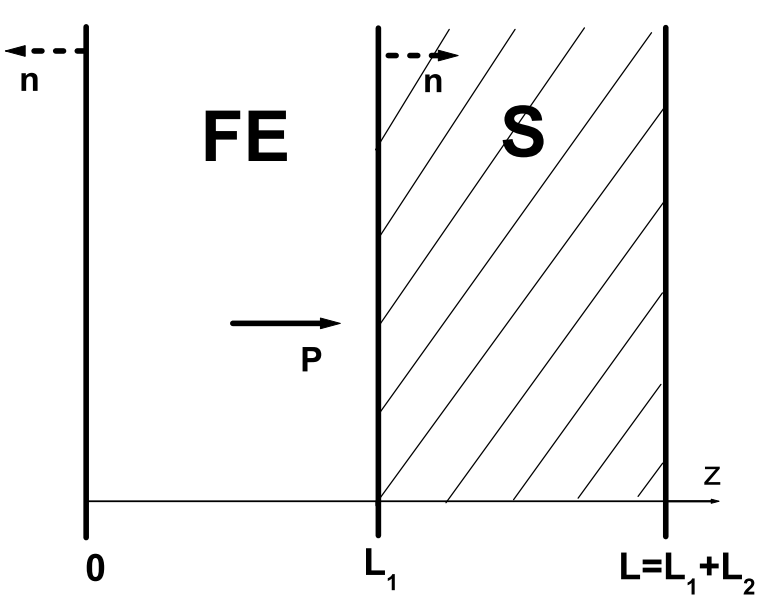

FIG. 1: Scheme of the periodic FE-S multilayer structure.

For the superconducting S-film without magnetic field the free energy can be written as

$F_{S}=\int_{L_{1}}^{L} d z\left[\frac{1}{2} a_{S}|\psi(z)|^{2}+\frac{1}{4} b_{S}|\psi(z)|^{4}+\frac{\hbar^{2}}{2 m e}\left|\frac{d \psi(z)}{d z}\right|^{2}\right]$,

where $a_{S}=a_{S}^{0}\left(T-T_{c}^{S}\right)$ with $T_{c}^{S}$ denoting the bulk superconducting transition temperature, and $m, e$ and $|\psi(z)|^{2}$ are the mass, charge and the local density of the Cooper pairs respectively. Similarly to (2) we assume in (3) that $\psi(\boldsymbol{r})$ depends on $z$ only, i.e., $\psi=\psi(z)$. Note also that without a magnetic field the phase $\phi=$ const, and the vector potential $A=\frac{\hbar c}{2 e} \frac{d \phi}{d z}=0$ (see the Appendix). The Ginzburg-Landau equations in this static case contain only the terms with $|\psi(z)|$ and below for simplicity we use the notations $|\psi|=\psi$.

The interface part of the free energy (1) describes the interaction between the spontaneous polarization and the superconducting charge near the interface. In order to derive $F_{\text {int }}$, we consider first the interaction of $P$ with an electric field $\boldsymbol{E}=(0,0, E(z))$ associated with the polarization distribution 25

$$
\begin{aligned}
& \int_{0}^{L_{1}} d z\left(-\frac{1}{2} \boldsymbol{P} \boldsymbol{E}+\frac{D(z) E(z)}{4 \pi}\right) \\
& =\frac{1}{2} \int_{0}^{L_{1}} d z P(z) E(z)+\frac{1}{4 \pi} \int_{0}^{L_{1}} E^{2}(z),
\end{aligned}
$$

, where $\boldsymbol{D}=\boldsymbol{E}+4 \pi \boldsymbol{P}=\varepsilon \boldsymbol{E}$ is the electric displacement. The field $\boldsymbol{E}$ can be determined from the Maxwell equation inside the FE-layer $\operatorname{div} \boldsymbol{D}=\operatorname{div}(\boldsymbol{E}+4 \pi \boldsymbol{P})=0$. The integration in the vicinity of the contacts gives us near $z=0$

$$
E(z)-E\left(0^{+}\right)=-4 \pi\left(P(z)-P\left(0^{+}\right)\right)
$$

and near $z=L_{1}$

$$
E\left(L_{1}^{-}\right)-E(z)=-4 \pi\left(P\left(L_{1}^{-}\right)-P(z)\right) .
$$


In contrast to the normal metal electrodes where $\boldsymbol{P}=0$ and $\boldsymbol{E}=0$, we employ the following two concepts for the FE-S interface: (i) $P\left(0^{-}\right), P\left(L_{1}^{+}\right) \neq 0$ (see the corresponding discussion in Introduction); (ii) an electric field can penetrate inside the superconductor $(E(z \geq$ $\left.\left.0^{-}\right), E\left(z \geq L_{1}^{+}\right) \neq 0\right)$ and thus substantially influence the superconducting properties $9.12,26$. Using these assumptions together with the boundary conditions for $D^{27}$

$$
\left(\left.\boldsymbol{D}\right|_{S}-\left.\boldsymbol{D}\right|_{F E}\right) \cdot \boldsymbol{n}=4 \pi \sigma,
$$

where $\sigma$ denotes the charge density induced by the polarization at the FE-S contact and $\boldsymbol{n}$ is the unit vector directed perpendicular to the surface of the ferroelectric into the S-layers, we obtain from (5) and (6)

$$
\begin{aligned}
& E\left(0^{-}\right)+4 \pi P\left(0^{-}\right)=-4 \pi \sigma\left(0^{-}\right), \\
& E\left(L_{1}^{+}\right)+4 \pi P\left(L_{1}^{+}\right)=4 \pi \sigma\left(L_{1}^{+}\right), \\
& E(z)=-4 \pi P(z), \quad 0^{+} \leq z \leq L_{1}^{-} .
\end{aligned}
$$

To derive the expressions for $E(z)$ penetrating inside the S-film at a distance of the Thomas-Fermi charge screening length $l_{T F}$, we consider the Poisson's equation near the right $\left(L_{1}<z<L_{1}+l_{T F}\right)$ and left $\left(-l_{T F}<z<0\right)$ FE-S contacts 27

$$
l_{T F}^{2} \frac{d^{2} \varphi}{d z^{2}}=\varphi
$$

where $-\nabla_{z} \varphi(z)=E(z)$, with the boundary conditions for the electrostatic potential $\varphi$

$$
-\nabla_{z} \varphi\left(L_{1}^{+}\right)=E\left(L_{1}^{+}\right), \quad-\nabla_{z} \varphi\left(0^{-}\right)=E\left(0^{-}\right) .
$$

Here $l_{T F}^{2}=\frac{\varepsilon_{F}}{6 \pi n_{0} e^{2}}$ is larger for systems with the lower mobile charge carrier concentration $n_{0}$, and $\varepsilon_{F}$ is the local Fermi energy. The solutions obtained from (9), (10) decay exponentially inside the right S-film $\left(z>L_{1}\right)$

$$
\varphi(z)=E\left(L_{1}^{+}\right) l_{T F} \cdot \mathrm{e}^{\frac{L_{1}-z}{l_{T F}}}, \quad E(z)=E\left(L_{1}^{+}\right) \mathrm{e}^{\frac{L_{1}-z}{l_{T F}}}
$$

and the left S-film $(z<0)$

$$
\varphi(z)=-E\left(0^{-}\right) l_{T F} \cdot \mathrm{e}^{\frac{z}{T F}}, \quad E(z)=E\left(0^{-}\right) \mathrm{e}^{\frac{z}{T F}} .
$$

The electric field penetration depth $l_{T F}$ in HTS compounds is shown to be in the range of about $0.5 \mathrm{~nm}$ (Ref. 10), which is significantly larger than $l_{T F} \sim 0.02 \mathrm{~nm}$ in the normal metal electrodes. Note that the total charge in the S-layer: $Q=\int_{L_{1}}^{L} d z \rho(z)=0$ includes the electron and background contributions: $\rho(z)=\rho_{b}(z)+$ $\rho_{e}(z)$ with the electron charge consisting of the normal and superconducting components: $\rho_{e}(z)=\rho_{e}^{n}(z)+\rho_{e}^{s}(z)$ according to the two-fluid model of superconductivity ${ }^{29}$. Since we study in this work the superconducting contribution $\rho_{e}^{s}(z)$, we assume here that the induced surface density $\sigma$ is described by a local increase/decrease of the superconducting charge density

$$
\begin{aligned}
& \sigma\left(L_{1}^{+}\right)=\chi_{0} \int_{L_{1}^{+}}^{L_{1}+l_{T F}}|\psi(z)|^{2} d z=\gamma_{0}\left|\psi\left(L_{1}^{+}\right)\right|^{2}, \\
& \sigma\left(0^{-}\right)=\gamma_{0}\left|\psi\left(L^{-}\right)\right|^{2},
\end{aligned}
$$

where $\gamma_{0} \sim \chi_{0} l_{T F}$ is the coupling coefficient characterizing the change of the charge carrier density near the contacts due to the spontaneous polarization in the FElayer.

From the expressions (8), (13), and (4) we obtain $F_{\text {int }}=F_{\text {int }}^{0}+F_{F E-S}$, where the interaction energy inside the FE-layer

$$
F_{i n t}^{0}=2 \pi \int_{0}^{L_{1}} d z P^{2}(z)
$$

The electrostatic interaction of the penetrating field $E(z)$ given by (11)-(12), with the superconducting charge $\rho_{e}^{s}$ near the contacts $\left(L_{1}<z<L_{1}+l_{T F}\right)$ and $\left(-l_{T F}<z<\right.$ $0)$ is determined by

$$
F_{F E-S}=\left(\int_{L_{1}}^{L_{1}+l_{T F}}+\int_{-l_{T F}}^{0}\right) d z \varphi(z)|\psi(z)|^{2} .
$$

To calculate the integrals in (15), we use the expansion of $\psi(z)$ at a small distance $\delta z<l_{T F} \ll L_{1}$ near the contact $z=L_{1}$

$$
\psi(z)=\psi\left(L_{1}^{+}\right)+\frac{d \psi\left(L_{1}^{+}\right)}{d z}\left(z-L_{1}\right)+\ldots
$$

and $z=0$

$$
\psi(z)=\psi\left(0^{-}\right)+\frac{d \psi\left(0^{-}\right)}{d z} z+\ldots,
$$

and the expressions (11) and (12) for $\varphi$. As the result, we get

$$
\begin{aligned}
& F_{F E-S}=-\frac{\gamma}{2} P\left(L_{1}^{+}\right)\left|\psi\left(L_{1}^{+}\right)\right|^{2}+\frac{\gamma}{2} P\left(0^{-}\right)\left|\psi\left(L^{-}\right)\right|^{2} \\
& +\delta\left(\left|\psi\left(L_{1}^{+}\right)\right|^{4}+\left|\psi\left(L^{-}\right)\right|^{4}\right)+2 \delta_{1}\left(P\left(L_{1}^{+}\right)\left|\psi\left(L_{1}^{+}\right)\right| \frac{d \psi\left(L_{1}^{+}\right)}{d z}\right. \\
& \left.-P\left(0^{-}\right)\left|\psi\left(0^{-}\right)\right| \frac{d \psi\left(0^{-}\right)}{d z}\right)+\mathcal{O}\left(\gamma^{2}\right),
\end{aligned}
$$

where $\gamma / 2=4 \pi(1-1 / \mathrm{e}) l_{T F}^{2}, \delta=4 \pi(1-1 / \mathrm{e}) \gamma_{0} l_{T F}^{2} \sim \gamma^{3 / 2}$ and $\delta_{1}=4 \pi(1-2 / \mathrm{e}) l_{T F}^{3} \sim \gamma^{3 / 2}$. The first term (14) renormalizes the coefficient $a_{F}$ in (2) and leads to a symmetrical reduction of the deviation of $P$ from its bulk value near the boundaries as was shown in Ref. 28. As a first step in the analysis of the role of the interface charge carriers, in this work we focus our attention on the contribution of the first two interface terms in the $F_{F E-S}$ given by (18), neglecting the smaller higher order terms in the $\gamma$-expansion and disregarding the well studied term (14). It should be noted that the contribution (18) can be derived from a microscopic model describing an Ising-type FE-layer sandwiched between the S-planes with BCS-pairing 24 . In the latter approach, the coupling energy $-\left.\frac{\partial \varepsilon l}{\partial R}\right|_{R=R_{0}}$ characterizes the change of the oneelectron energy $\varepsilon_{l}$ in the S-plane due to the distortion of the nearest atomic group of the FE-layer with the coordinate $R=R_{0}+\Delta_{R}$ (the average distortion $\Delta_{R}$ becomes nonzero in the ferroelectric state). 


\section{A. The Ginzburg-Landau equations}

Minimization of the total free energy $f=\int_{\Omega} d \Omega\left(F_{F E}+\right.$ $\left.F_{S}+F_{F E-S}\right)$ where $\Omega$ denotes the $(x, y)$-surface, by variation of the order parameters $P$ and $\psi$ yields the following two Ginzburg-Landau equations

$$
\begin{array}{ll}
a_{F} P(z)+b_{F} P^{3}(z)-c_{F} \frac{d^{2} P(z)}{d z^{2}}=0, & 0<z<L_{1}(19) \\
a_{S} \psi(z)+b_{S} \psi^{3}(z)-\frac{\hbar^{2}}{m e} \frac{d^{2} \psi(z)}{d z^{2}}=0, & L_{1}<z<L(20)
\end{array}
$$

The extra surface terms appearing from the variation, give the set of the boundary conditions at the right contact $z=L_{1}^{+}$

$$
\begin{aligned}
& c_{F} \frac{d P\left(L_{1}^{+}\right)}{d z}-\frac{\gamma}{2} \psi^{2}\left(L_{1}^{+}\right)=0, \\
& \frac{\hbar^{2}}{m e} \frac{d \psi\left(L_{1}^{+}\right)}{d z}+\gamma \psi\left(L_{1}^{+}\right) P\left(L_{1}^{+}\right)=0,
\end{aligned}
$$

and at the left contact $z=0^{-}\left(z=L^{-}\right)$

$$
\begin{aligned}
& c_{F} \frac{d P\left(0^{-}\right)}{d z}-\frac{\gamma}{2} \psi^{2}\left(L^{-}\right)=0, \\
& \frac{\hbar^{2}}{m e} \frac{d \psi\left(L^{-}\right)}{d z}+\gamma \psi\left(L^{-}\right) P\left(0^{-}\right)=0 .
\end{aligned}
$$

Setting $\gamma=0$ in (21) and (22) results in ordinary free boundary conditions (zero gradients of the order parameters) at the FE-S contacts, which implies in fact the continuity of $P$ and $\psi$ near the boundaries. However, a small finite coupling energy $\gamma \neq 0$ gives non-zero gradients of $P$ and $\psi$

$$
\begin{aligned}
& \frac{d P_{c}}{d z} \sim \gamma \psi_{c}^{2}, \\
& \frac{d \psi_{c}}{d z} \sim-\gamma \psi_{c} P_{c},
\end{aligned}
$$

where $\psi_{c}$ and $P_{c}$ denote the corresponding contact values.

Consider the first expression (23) for $P$. As the right hand side is always positive, we conclude that

$$
\frac{d P_{c}}{d z}>0
$$

For $P>0$ this relation gives at the right contact $z=L_{1}$ an increase of $P$ due to the coupling with the surface charge, whereas at the left contact $z=0, P$ decreases. Since for $P<0$ we have the similar behavior, we conclude that independent of the direction of the polarization

$$
P\left(z=L_{1}\right)>P(z=0) .
$$

Let us analyze the second condition (23) for $\psi$ which in distinction to the latter case depends on the direction of the polarization. For $P>0$, the gradient $\frac{d \psi_{c}}{d z}<0$ and we obtain an increase of the electronic pair density at $z=L_{1}$ and a lower density at $z=L$. Furthermore, a switching of the polarization $(P<0)$ results in $\frac{d \psi_{c}}{d z}>0$ and in the opposite behavior of the charge density at the contacts.

Below we present the numerical results for the problem (19)-(22) in the case when $T_{c}^{F}<T_{c}^{S}$, discussing in limiting cases the analytical solutions for $P$ and $\psi$. Note that the case $T_{c}^{F}<T_{c}^{S}$ is realized in complex insulating perovskite alloys such as BST with high Sr content. The ferroelectric transition temperature in BST depends on the Ba content $x$, approaching $T_{c}^{F} \approx 0$ for $x=0\left(\mathrm{SrTiO}_{3}\right.$ is a paraelectric material with no ferroelectric phase transition) and $T_{c}^{F}=120^{\circ} \mathrm{C}$ for $x=1\left(\mathrm{BaTiO}_{3}\right)$. For instance, for $7.5 \% \mathrm{Ba}, T_{c}^{F}=60 \mathrm{~K}^{12}$ which lies already in the range of superconducting transition temperatures of the HTS compounds.

\section{CASE STUDY: $T_{c}^{F}<T_{c}^{S}$}

We now present a brief overview of the results obtained for our model (19)-(22) for different temperatures and coupling $\gamma$. Fig. 2 shows spatial dependences of $P$ and $\psi$ normalized by the zero-temperature values $P_{0}=\sqrt{a_{F}^{0} / b_{F}}$ and $\psi_{0}=\sqrt{a_{S}^{0} / b_{S}}$ for the following set of the parameters: $\tilde{\eta}=\eta \frac{\psi_{0}^{2} \tilde{a}_{S}^{0}}{P_{0}^{2}}=0.2\left(\eta=\xi_{S}^{0} / \xi_{F}^{0}\right.$, $\left.\tilde{a}_{S}^{0}=a_{S}^{0} / a_{F}^{0}\right)$ and $\tilde{\gamma}=\frac{\gamma}{a_{F}^{0} T_{c}^{F}} \frac{\psi_{0}^{2}}{\xi_{F}^{0} P_{0}}=0.1$ at a low temperature $\tau=T / T_{c}^{F}=0.5$, while $\tau_{c}^{S}=T_{c}^{S} / T_{c}^{F}=1.3$. Here $\xi_{S}^{0}=\sqrt{\frac{\hbar^{2}}{2 m e a_{S}^{0} T_{c}^{S}}}$ is the zero-temperature superconducting coherence length and the correlation length for the FE-layer at $T=0$ is: $\xi_{F}^{0}=\sqrt{\frac{c_{F}}{a_{F}^{0} T_{c}^{F}}}$. Note that taking into account the possible variation of the field penetration depth (section III), we can estimate the range for the magnitude of the dimensionless coupling $\tilde{\gamma}$ : from $\sim 10^{-2}$ (for conventional superconductors) to $\sim 1$ (for HTS films). For the films of moderate thickness $\left(L_{1} / \xi_{F}^{0}=1\right.$ and $\left.L_{2} / \xi_{S}^{0}=1\right)$, one can observe a strong coupling effect demonstrated in Fig. 2] Similarly to the theory for bulk ferroelectrics, the two possible directions of the polarization can be stabilized in the FElayer. As was already discussed in section $\llbracket A$ (equations (23) and (24)), $P$ increases at the right contact, $z=L_{1}$, and lowers at the left one due to the coupling with the Selectrons (Fig. 22(a)). The important effect arising from this, is the appearance of two stable solutions for $\psi$ in the adjacent S-layer (Fig. 2(b)). The first of them (with $\left.d \psi / d z<0, \psi\left(L_{1}\right)>\psi(L)\right)$ corresponds to the positive branch of $P$ whereas the second one $\left(\psi\left(L_{1}\right)<\psi(L)\right)$ appears due to the coupling with the negative $P$. Note that the regions with the lower density $|\psi|^{2}$ (the depletion layer) and the accumulation charge are located near the contacts $\left(L-\delta_{S}<z<L\right)$ and $\left(L_{1}<z<L_{1}+\delta_{S}\right)$ with $\delta_{S} \sim \xi_{S}=\sqrt{\left|\frac{\hbar^{2}}{2 m e a_{S}}\right|}$, whereas inside the thick S-layer (Fig. 2(b), inset), $\psi$ approaches its bulk value $\psi_{b}=\sqrt{-\frac{a_{S}}{b_{S}}}$. 

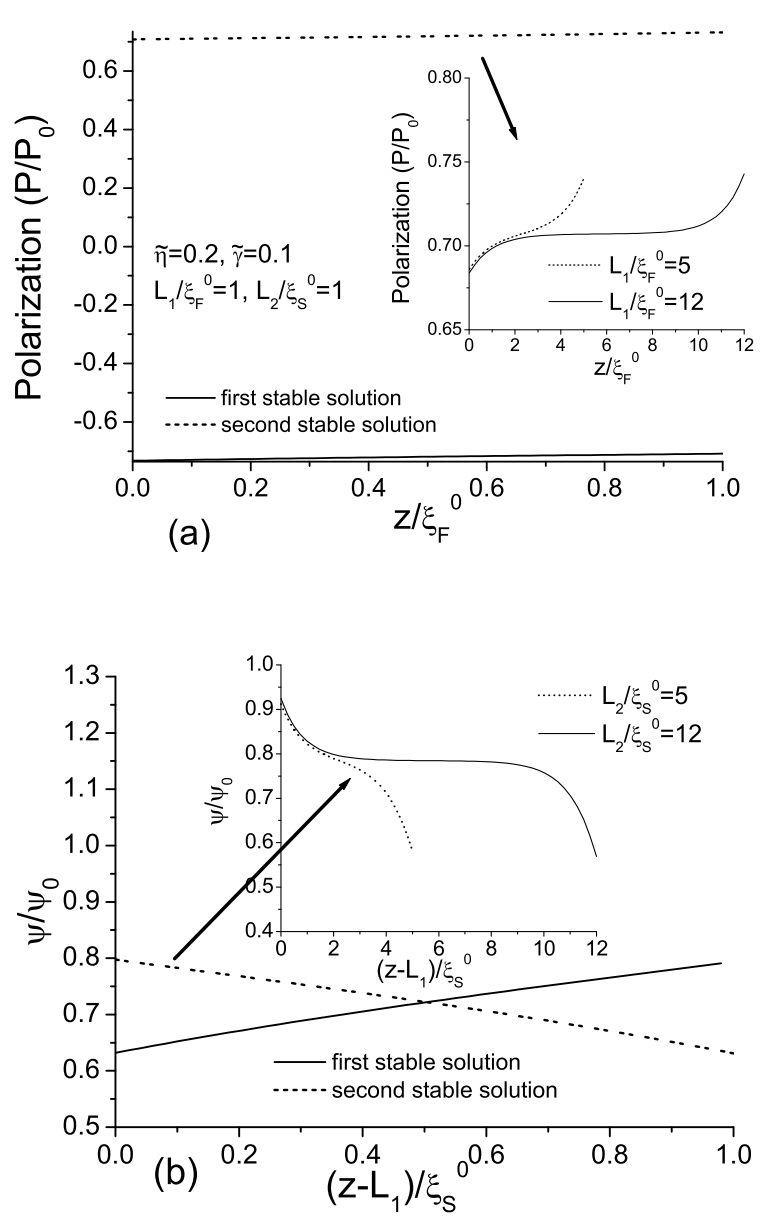

FIG. 2: Spatial profiles of (a) $P$ and (b) $\psi$ in the ordered $\mathrm{FE}+\mathrm{SC}$ phase at $\tau=0.5<\tau_{c}^{F}=1$ exhibiting the ferroelectric ordering in a FE-film and the superconductivity in a S-film of moderate thicknesses $\left(L_{1} / \xi_{F}^{0}=1\right.$ and $\left.L_{2} / \xi_{S}^{0}=1\right)$. The insets demonstrate the corresponding profiles for thick layers.

Similar interface effects with an increase of the FElayer thickness are demonstrated in the insets of Fig. 2(a), where the regions of the deviations of $P$ from its bulk value $P_{b}=\sqrt{-\frac{a_{F}}{b_{F}}}$ are located within a distance $\delta_{F} \sim$ $\xi_{F}=\sqrt{\left|\frac{c_{F}}{a_{F}}\right|}$ from the interfaces.

The whole physical behavior becomes highly nontrivial for $T>T_{c}^{F}(\tau>1)$ in the temperature range $\tau_{c}^{F}<\tau<$ $\tau_{c}^{S}$.

\section{A. The phase with symmetric domains}

In contrast to the bulk, where $P=0$ for $T>T_{c}^{F}$, the coupling with the interface charge leads to rather unusual behavior for $P$ and $\psi$ as shown in Fig. 3 at $\tau=1.1$. The polarization in this case is enhanced, and for $T_{c}^{F}<T<$
$T_{c}^{S}$ and small $\tilde{\gamma}<1$ is given by

$$
P(z)=\frac{\gamma}{2 c_{F}} \xi_{F} \frac{\sinh \left(z-\frac{L_{1}}{2}\right) / \xi_{F}}{\cosh \frac{L_{1}}{2 \xi_{F}}}\left|\psi\left(L^{-}\right)\right|^{2},
$$

where the superconducting pair density at $z=L^{-}$

$$
\left|\psi\left(L^{-}\right)\right|^{2}=\psi_{b}^{2}\left[1+\frac{\gamma^{2}}{c_{F} b_{S}} \frac{\xi_{F}}{\xi_{S}} \tanh \frac{L_{1}}{2 \xi_{F}} \cot \frac{L_{2}}{2 \xi_{S}}\right]+\mathcal{O}\left(\gamma^{4}\right) .
$$

As follows from (26), in this state a non-zero $P$ exists only due to the superconducting electrons, because setting $\gamma=0$ or $\psi(L)=0$ immediately yields $P=0$.

For the thin FE-films with $\frac{L_{1}}{\xi_{F}}<1$ or close to the bulk transition temperature (where $\xi_{F} \rightarrow \infty$ ), the effect of the contacts is particularly pronounced and the polarization is linear in $z$

$$
P(z)=\frac{\gamma}{2 c_{F}}\left(z-\frac{L_{1}}{2}\right)|\psi(L)|^{2} .
$$

However, as the FE-layer becomes thicker $\left(\frac{L_{1}}{\xi_{F}} \gg 1\right)$, the polarization deviates from zero only near the interfaces, whereas inside the layer $P \rightarrow 0$. For example, for $z \approx 0$

$$
P \sim-\gamma \xi_{F} \psi_{b}^{2} e^{-z / \xi_{F}}
$$

and decays exponentially as one moves towards the interior of the layer. Near the right contact $z \approx L_{1}$, the behavior is similar

$$
P \sim \gamma \xi_{F} \psi_{b}^{2} e^{-\left(L_{1}-z\right) / \xi_{F}} .
$$

Note that the polarization is antisymmetric with respect to the center of the FE-layer $z=L_{1} / 2: P(z)=$ $-P\left(L_{1}-z\right)$, forming two $P$-domains: with a negative $P$ for $0 \leq z \leq L_{1} / 2$, and a positive $P$ for $L_{1} / 2 \leq z \leq L_{1}$, as shown in Fig. 3(a). The center of the layer, where $P\left(z=L_{1} / 2\right)=0$, corresponds to the domain wall.

Consider now the behavior of the S-layer. For thin S-films $\left(\frac{L_{2}}{\xi_{S}}<1, L_{2} \neq 0\right)$ in the vicinity of the $T_{c}^{F}$ $\left(T_{c}^{F}<T<T_{c}^{S}\right)$, and for a weak coupling $\tilde{\gamma}<1$, the superconducting order parameter is given by

$\psi(z)=\psi_{b}\left[1+\frac{\gamma^{2}}{2 c_{F} b_{S} \eta} \tanh \frac{L_{1}}{2 \xi_{F}} \frac{\cos \frac{z-L_{1}-L_{2} / 2}{\xi_{S}}}{\sin \frac{L_{2}}{2 \xi_{S}}}\right]+\mathcal{O}\left(\gamma^{4}\right)$.

In contrast to the antisymmetric behavior of $P$ in the domain-type phase, $\psi(z)$ is symmetric about the center of the S-layer $z=L_{1}+L_{2} / 2: \psi\left(L_{1}+z\right)=\psi(L-z)$ as can be seen in Fig. [3 (b). We note that for the case of thin S- or FE-films $\left(L_{1} / \xi_{F} \ll 1\right.$ or $\left.L_{2} / \xi_{S} \ll 1\right)$ the effect of fluctuations is expected to be essentially important. This can be studied by including the additional higher-order terms into the free-energy functional (2)-(3) that will be a subject of separate work. From (31) it follows immediately that as $\gamma \rightarrow 0$ or $\eta=\frac{\xi_{S}}{\xi_{F}} \rightarrow \infty, \psi(z)$ approaches 

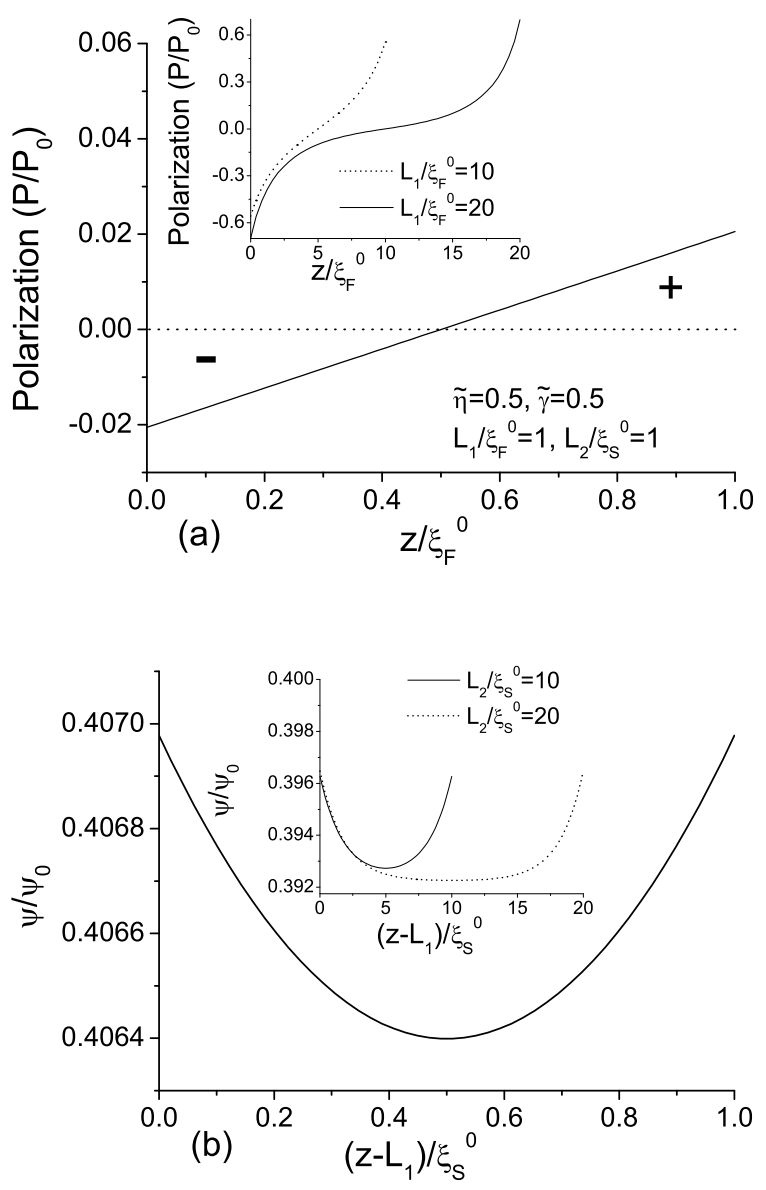

FIG. 3: Spatial profiles of (a) $P$ and (b) $\psi$ in the domain phase at $\tau=1.1>\tau_{c}^{F}$ exhibiting the weak ferroelecity in a thin FE-film and the superconductivity in a thin S-layer $\left(L_{1} / \xi_{F}^{0}=1\right.$ and $\left.L_{2} / \xi_{S}^{0}=1\right)$. The insets show the corresponding profiles for thick layers.

its bulk value $\psi_{b}$. Moreover, we obtain the bulk behavior in (31) also when the adjacent FE-film becomes thin, so that $L_{1} \ll \xi_{F}$. Similarly to the FE-layer, in a thick S-layer the influence of the interfaces is remarkable only near the contacts (Fig. B(b), inset).

To gain a better understanding of the nature of the domain phase, we determine its free energy which can be obtained by the substitution of $P$ and $\psi$ from (26) and (31) into (21), (3) and (18)

$$
F_{d m n}=-\frac{\gamma^{2}}{4 c_{F}} \xi_{F} \psi_{b}^{4} \tanh \frac{L_{1}}{2 \xi_{F}}-L_{2} \frac{b_{S}}{4} \psi_{b}^{4}+\mathcal{O}\left(\gamma^{4}\right) .
$$

Note that since for the free (non-coupled) FE-layer $F_{F E}^{0}=0$ for $T>T_{c}^{F}$, the total free energy for the noncoupled $\mathrm{FE}+\mathrm{S}$ multilayers for $T_{c}^{F}<T<T_{c}^{S}$ is given by

$$
F_{F E+S}^{0}=-L_{2} \frac{b_{S}}{4} \psi_{b}^{4}
$$

Therefore, the domain state always has an energy gain due to a negative difference between the energies in these two phases

$$
\Delta F_{d m n}=F_{d m n}-F_{F E+S}^{0}=-\frac{\gamma^{2}}{4 c_{F}} \xi_{F} \psi_{b}^{4} \tanh \frac{L_{1}}{2 \xi_{F}}+\mathcal{O}\left(\gamma^{4}\right) .
$$

The latter result supports our conclusion that a non-zero coupling with $\psi \neq 0$ is the main reason for the stabilization of the domain state. We note also that as $\gamma \rightarrow 0$, we obtain that the energy gain $\Delta F_{d m n} \rightarrow 0$, the polarization $P \rightarrow 0$, and hence the domain phase transforms in fact into an ordinary paraelectric state:

$$
T_{c}^{F, \gamma} \rightarrow T_{c}^{F}, \quad \text { as } \quad \gamma \rightarrow 0 .
$$

Thus in the following analysis we define $T_{c}^{F, \gamma}$ as the temperature of the transition between the ordered $\mathrm{FE}+\mathrm{SC}$ phase with the bulk-type $P \neq 0$, and the domain-type phase, which occurs on heating.

\section{B. Strong coupling behavior}

As results from the previous section, generally a weak coupling $\tilde{\gamma} \ll 1$ at the contacts produces symmetric domain phase with a non-zero polarization for $T_{c}^{F}<T<$ $T_{c}^{S}$. We demonstrate how this behavior changes as the coupling becomes stronger, i.e., with the increasing $\tilde{\gamma}$. In Fig. 团 we plot the order parameters vs $\tilde{\gamma}$ for layers of thicknesses $L_{1} / \xi_{F}^{0}=2$ and $L_{2} / \xi_{S}^{0}=2$. At lower $\tau$ ( $\tau=0.5$ and $\tau=0.55$ ), the behavior of $\psi$ and $P$ rapidly changes at $\tilde{\gamma} \approx 0.33$, corresponding to the transition to the normal (non-superconducting) state $(\psi=0)$. In this state, $P=P_{b}=$ const does not depend on $z$ because setting $\psi=0$ in (22) and (21) gives the free boundary conditions. The suppression of the superconductivity due to the strong contact with polarization resembles the effect of an external field, impurities and other pair-breaking factors on superconductors ${ }^{29}$. Like these factors, the polarization at the boundaries with the S-layer supplies additional energy (18) for the breaking of electron pairs in the strong-coupling regime.

From the point of view of the Landau-Ginzburg functional (2), (3), (18), an increase of $\gamma$ induces larger values of the fourth-order fluctuation terms as well as the third terms in (21) and (3) describing the spatial inhomogeneities, and hence drives a transition to the homogeneous state with $\psi=0$ which has the lower energy. To explain qualitatively the effect of $\gamma$, we consider for simplicity only the left FE-S contact with the energy $\frac{\gamma}{2} P\left(0^{-}\right) \psi^{2}\left(L^{-}\right)$assuming at the right contact the free boundary conditions. At low $T$ the expression for the free energy in the ordered $\mathrm{FE}+\mathrm{S}$-phase is given by

$$
F_{F E+S} \approx-\frac{1}{4}\left[P_{b}^{4} b_{F} L_{1}+\psi_{b}^{4} b_{S} L_{2}\right]+\frac{\gamma}{2} P_{b} \psi_{b}^{2} .
$$

The critical value for $\gamma$ can be found by equating (35) and the free energy for the state with the suppressed 
superconductivity

$$
F_{\text {suppr }}=-\frac{1}{4} P_{b}^{4} b_{F} L_{1}
$$

which yields

$$
\tilde{\gamma}_{c}=\frac{\psi_{b}^{2}}{2 P_{b}^{2}} \frac{\tilde{a}_{0}^{S}}{T_{c}^{F}} \eta \frac{L_{2}}{\xi_{S}^{0}}
$$

From (37) it follows, that as the S-layer becomes thicker, a stronger critical coupling is required to destroy the superconducting state. In contrast, a larger polarization $P$ in Eq. (37) reduces $\tilde{\gamma}_{c}$, which supports our interpretation of the analogy with other external field pair breaking effects.

Consider now the $\gamma$-dependence at higher temperatures shown in the insets of Fig. 目(a) and Fig. 世(b) slightly below $(\tau=0.9)$ and above $(\tau=1.15$, where the system for small $\tilde{\gamma}$ is in the domain phase) the bulk value $\tau_{c}^{F}=1$. Like at low temperatures, the superconductivity of the domain phase $(\tau=1.15)$ is destroyed when approaching the critical value $\tilde{\gamma}_{c}^{e} \approx 0.55$. We note that since $\psi=0$ for $\tilde{\gamma}>\tilde{\gamma}_{c}^{e}$, the polarization, which is enhanced here only due to the coupling with $\psi$, vanishes and we obtain the combined paraelectric + suppressed superconducting $(P=0, \psi=0)$ phase for $\tau>\tau_{c}^{F}$. We emphasize that in contrast to the bulk ferroelectrics exhibiting $P=0$ in this temperature range, in our coupled FE-layer the zero polarization is only possible due to the strong coupling effect depressing the source for the enhancement of $P$.

The behavior for large $\tilde{\gamma}$ can be even more complicated as demonstrated in the inset of Fig. 4 at the temperature $\tau=0.9$ which is below but close to $\tau_{c}^{F}$ (Fig. 廿(a)). Despite $\tau<\tau_{c}^{F}$, the $\gamma$-dependence of $P$ and $\psi$ clearly shows the stabilization of the domain state with $P(0)=-P\left(L_{1}\right)$ and $\psi\left(L_{1}\right)=\psi(L)$ in the range $(0.34<\tilde{\gamma}<0.46)$, and only with the further $\tilde{\gamma}$ increase $(\tilde{\gamma}>0.46)$, the transition to the $\left(P=P_{b}, \psi=0\right)$-state. To analyze more comprehensively the behavior for different $\tilde{\gamma}$, we discuss in the next section the phase diagrams $(\tilde{\gamma}, \tau)$ plotted for the different FE- and S-layer thicknesses.

\section{PHASE DIAGRAMS $(\tilde{\gamma}, \tau)$}

The phase diagrams $(\tilde{\gamma}, \tau)$ plotted in Fig. 5 and Fig. 6 show the regions of stability for different phases discussed in section III As has been revealed in the foregoing analysis, the following phases can exist in our periodical multi-layer system: (i) the ordered bulk-type FE+SC phase with the nonzero spontaneous polarization in the FE-layer and the superconductivity in the S-layer; (ii) the superconductivity in the S-layer coexisting with the ferroelectric domain state exhibiting the symmetric (negative and positive) polarization domains due to the FE-S contacts (so called domain-type phase); (iii) the phase
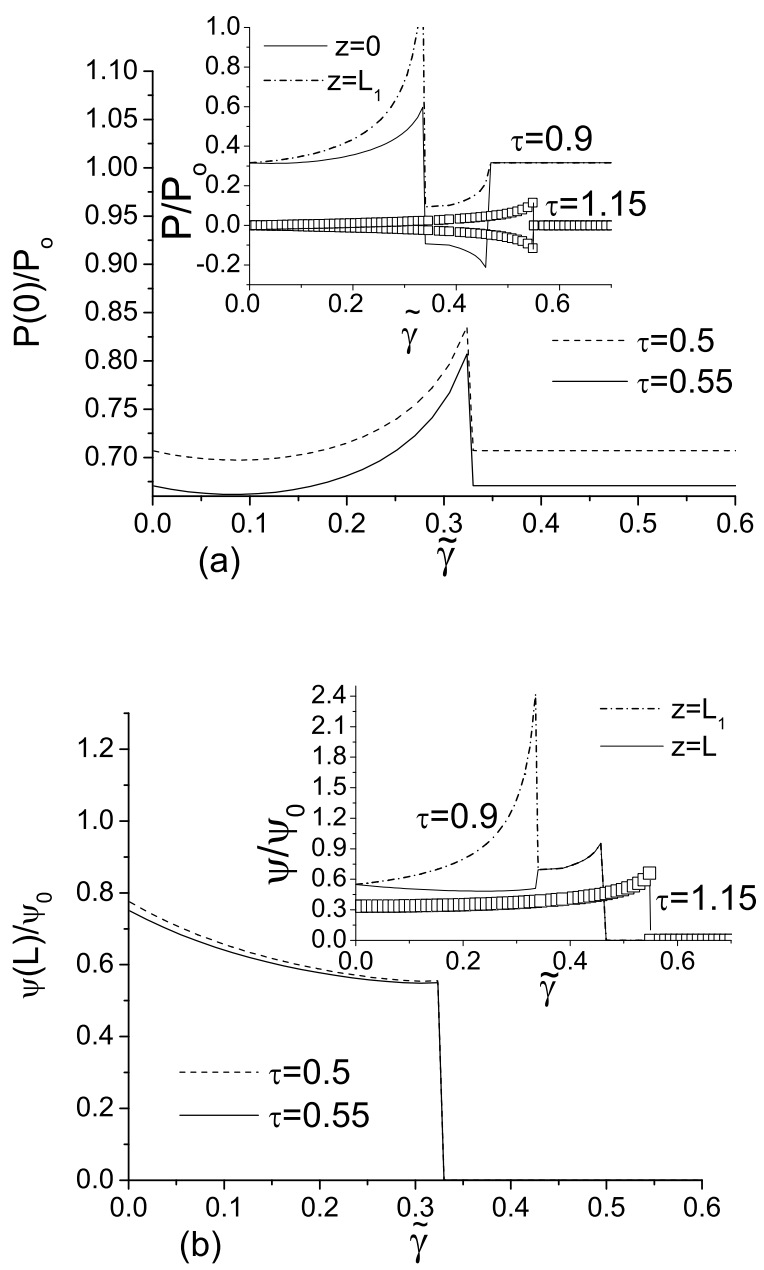

FIG. 4: (a) Polarization in the FE-film of thickness $L_{1} / \xi_{F}^{0}=2$ and (b) $\psi$ at $z=L$ in the S-film of thickness $L_{2} / \xi_{S}^{0}=2$ vs the magnitude of the coupling $\tilde{\gamma}$, exhibiting a transition from the FE+SC phase to the FE-state with suppressed superconductivity. Here $\tilde{\eta}=0.5$. The insets show the $P$ and $\psi$ behavior at the FE-S contacts with increasing $\tilde{\gamma}$ for $\tau=0.9$ (solid curves and dashes) and $\tau=1.15$ (open squares) with the stabilization of the domain state for $\tilde{\gamma}<\tilde{\gamma}_{c}^{e}$.

with the ferroelectricity and the suppressed superconductivity $(\mathrm{FE}+\mathrm{NM})$; (iv) the high-temperature paraelectric (in the FE-layer)+normal state (in the S-layer).

In Fig. 5 we plot a set of the diagrams for the S-film of moderate thickness $L_{2} / \xi_{S}^{0}=1$ coupled to the FElayers of different thickness: (a) a thin FE-film, $L_{1} / \xi_{F}^{0}=$ $0.3,1.0$, (b) a FE-layer of moderate thickness $L_{1} / \xi_{F}^{0}=$ 1.5 , and (c) a thick FE-layer with $L_{1} / \xi_{F}^{0}=2.5,5.0$. We associate in the following analysis the variation of the FEthickness as related to that of the S-layer, to the ratio

$$
\zeta=\frac{L_{1}}{L_{2}}=\eta^{-1} \frac{L_{1} / \xi_{F}^{0}}{L_{2} / \xi_{S}^{0}}
$$

where the ratio between the superconductor and the fer- 
roelectric coherence lengths $\eta$ ranges from $\sim 0.05$ (for HTS compounds) to $\sim 10$ (conventional superconductors), taking the typical value for $\xi_{F}^{0} \approx 10 \mathrm{~nm}$ (Ref. 30). As $\zeta$ decreases from $5 / \eta$ (Fig. 國(c)) to $0.3 / \eta$ (Fig. 5(a)), the area of the domain-type phase drastically expands towards larger $\tilde{\gamma}$ and lower $T$ and finally approaches even the low-temperature range as represented by arrows in Fig. 5(a). The expansion of the domain phase is accompanied by the simultaneous decrease of the region of the $\mathrm{FE}+\mathrm{NM}$ (suppressed)-phase. We note that in the system with a thicker FE-layer $\left(L_{1} / \xi_{F}^{0}=1\right.$, Fig. 国(a)), the transition to the suppressed state occurs at a significantly lower $\gamma_{c}^{e}\left(\tilde{\gamma}_{c}^{e} \approx 0.9\right)$ as compared to a thin FE-film (case $L_{1} / \xi_{F}^{0}=0.3$ in Fig. 囵(a)) with $\tilde{\gamma}_{c}^{e} \approx 1.8(\tau=1.1)$, which clearly demonstrates the crucial role of the ferroelectric layer in the suppression of the superconductivity. These modifications of the phase diagram can be explained by the competition between the first tendency to destroy the superconductivity prevailing for the coupling with thicker FE-layers, and the second tendency to retain the superconducting state and stabilize $P$-domains dominating in systems with thicker S-layers. As is demonstrated in Fig. [5 the decrease of $\zeta$ results in a dominant role of the S-layer and, as a consequence, in the enhancement of the region with $\psi \neq 0$ at lower $T<T_{c}^{F}$ (which in turn induces $P$-domains in the FE-layer). Since in the HTS-films $\eta$ is about three orders of magnitude smaller than in conventional superconductors, one might expect from (38) that even a slight variation of the S- or FE-film thickness leads to a dramatic modification of the phase diagram and, consequently, of the stable state of this type of compounds.

Fig. [6] shows the behavior of the system with a fixed FE-thickness $L_{1} / \xi_{F}^{0}=1$ and with a S-thickness $L_{2} / \xi_{S}^{0}$ varying from 15 (Fig. [6) (a)) to 0.2 (Fig. 6) (b)). As in the previous case, we find here the same tendency of expansion of the domain phase with decreasing $\zeta$. We also bring to attention that the destruction of the superconducting state demonstrated here has been observed in the measurements of the resistivity in the film $\mathrm{YBCO} / \mathrm{BaTiO}_{3}$ composites 31 .

\section{A. Comparison with the microscopic model of Ref. 24}

As has already been mentioned in section [II when the ferroelectric critical temperature $T_{c}^{F}$ is close to $T_{c}^{S}$, the phenomenological approach proposed in this work, has common key aspects with the microscopic description proposed in Ref. 24. However, while the latter model describes the superconducting layer as two boundary 2-D planes exhibiting BCS-superconductivity, the present approach allows us to study the behavior inside the thick Slayers. Despite the fact that the microscopic BCS-model of Ref. 24 does not consider directly the internal processes in the superconducting layer and treats the inner part of the layer as a source for the electron transfer to-
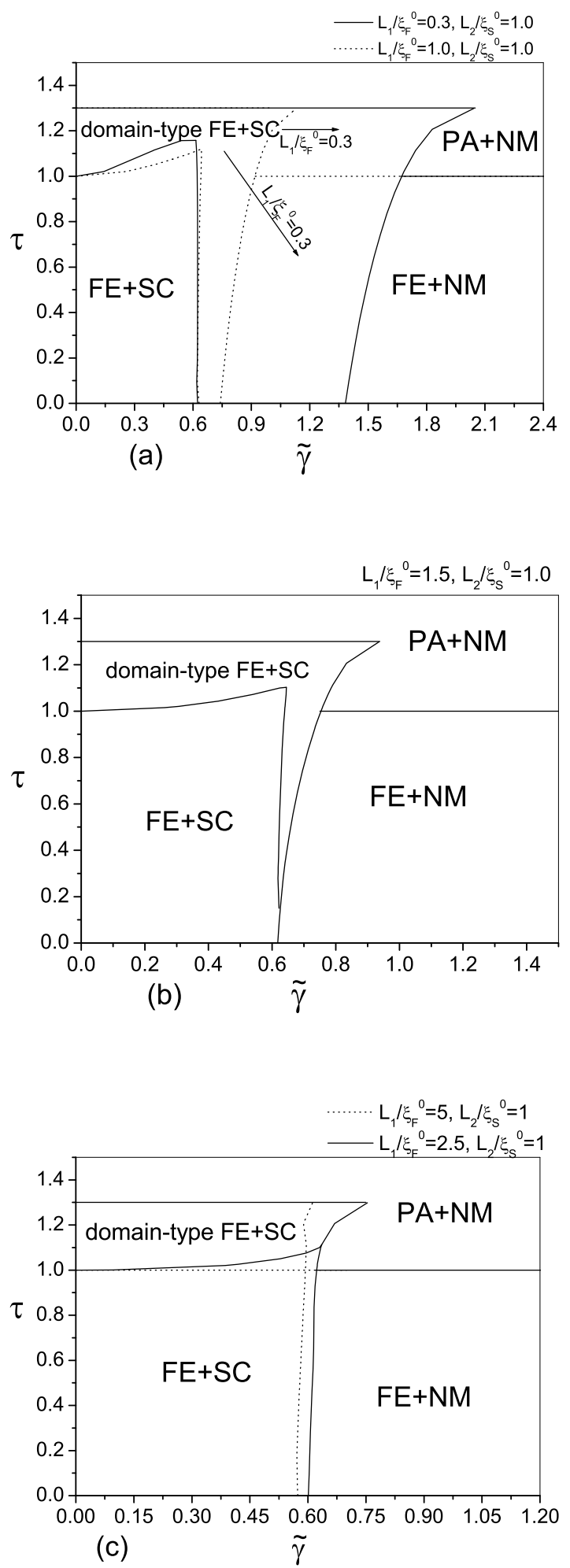

FIG. 5: Phase diagrams $(\tilde{\gamma}, \tau)$ for a compound containing S-films of thickness $L_{2} / \xi_{S}^{0}=1.0$ coupled to the FE-layers of thickness: (a) $L_{1} / \xi_{F}^{0}=0.3$ and $L_{1} / \xi_{F}^{0}=1.0$ (thin FEfilms); (b) $L_{1} / \xi_{F}^{0}=1.5$ (moderately thick FE-film) and (c) $L_{1} / \xi_{F}^{0}=2.5,5.0$ (thick FE-layer). Here $\tilde{\eta}=0.5$. 

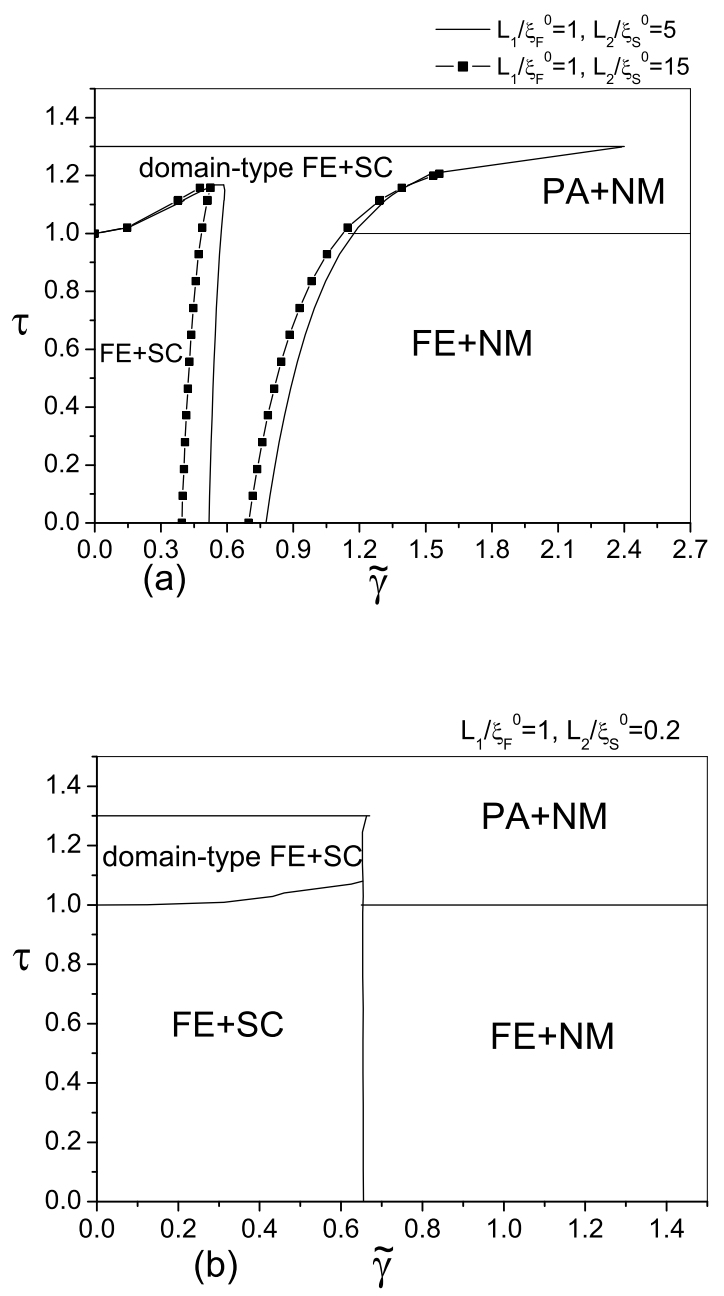

FIG. 6: Phase diagrams $(\tilde{\gamma}, \tau)$ for a compound containing FEfilms of thickness $L_{1} / \xi_{F}^{0}=1.0$ coupled to S-layers of thickness: (a) $L_{2} / \xi_{S}^{0}=5.0$ and $L_{2} / \xi_{S}^{0}=15.0$ (thick S-layer) and (b) $L_{2} / \xi_{S}^{0}=0.2$ (thin S-film). Here $\tilde{\eta}=0.5$.

wards (away from) the boundary S-planes, the following key results appear in both approaches:

(i) the enhancement of the ferroelectric polarization with a formation of positive and negative $P$-domains above the ferroelectric transition temperature as well as for large $\gamma$ and low temperatures;

(ii) the suppression of the superconductivity for the strong FE-S coupling due to the ferroelectric field-type effect.

Thus, the basic structure of the phase diagrams $(\gamma, T)$ together with the behavior of the system in different observed phases discussed in Ref. 24, also retains its fundamental features in the present phenomenological approach.

\section{B. Dependence of $T_{c}^{F, \gamma}$ on the FE- and S-layer thicknesses}

The behavior of the different phases discussed above with the variation of the FE- and S-thicknesses results in different types (from monotonous to complex nonmonotonic) of the corresponding $T_{c}^{F, \gamma}$ dependences.

Since the system properties drastically change for the large coupling $\tilde{\gamma}>\tilde{\gamma}_{c}$, the $L_{1}$ - and $L_{2}$-dependence of $T_{c}^{F, \gamma}$ is also qualitatively different in the weak $\left(\tilde{\gamma}<\tilde{\gamma}_{c}\right)$ and in the strong coupling regime $\left(\tilde{\gamma}>\tilde{\gamma}_{c}\right)$. To illustrate this, in Fig. ?? and Fig. ?? we plot several curves for various values of $\tilde{\gamma}$. Specifically, Fig. ??(inset) and Fig. ??(a) show the case $\tilde{\gamma}<\tilde{\gamma}_{c}$, whereas $\tilde{\gamma}>\tilde{\gamma}_{c}$ is represented by Fig. ??( $(\tilde{\gamma} \geq 0.9)$ and Fig. ??(b).

Depending on the value of $\tilde{\gamma}$, we can obtain the following characteristic types of $T_{c}^{F, \gamma}\left(L_{1}\right)$ and $T_{c}^{F, \gamma}\left(L_{2}\right)$ behavior:

(1) for $\tilde{\gamma}<\tilde{\gamma}_{c}, \tau_{c}^{F, \gamma}=T_{c}^{F, \gamma} / T_{c}^{F}$ decays monotonically with increasing $L_{1}$ to the bulk value $\tau_{c}^{F}=1$ (Fig. ??, $\tilde{\gamma}=0.1$ in the inset). We note that in contrast to the model in Ref. 24 where $T_{c}^{F, \gamma}$ lies slightly below $T_{c}^{F, b u l k}$, the coupling with a S-layer of a finite thickness produces here the enhancement of the bulk-type FE+SC-phase. This corresponds to the increase of $T_{c}^{F, \gamma}$ $\left(T_{c}^{F}<T_{c}^{F, \gamma}<T_{c}^{S}\right)$ for $\gamma \neq 0\left(\tilde{\gamma}<\tilde{\gamma}_{c}\right)$, observed also in the phase diagrams in Figs. 5 and 6 As a result, we also obtain that:

(2) for $\tilde{\gamma}<\tilde{\gamma}_{c}$, the increase of $L_{2}$ produces a monotonic increase of $\tau_{c}^{F, \gamma}$, with finally approaching a saturation value which is the higher the larger $\tilde{\gamma}$ (Fig. ??(a)).

(3) For $\tilde{\gamma}>\tilde{\gamma}_{c}$, the complex behavior of $\tau_{c}^{F, \gamma}$ vs

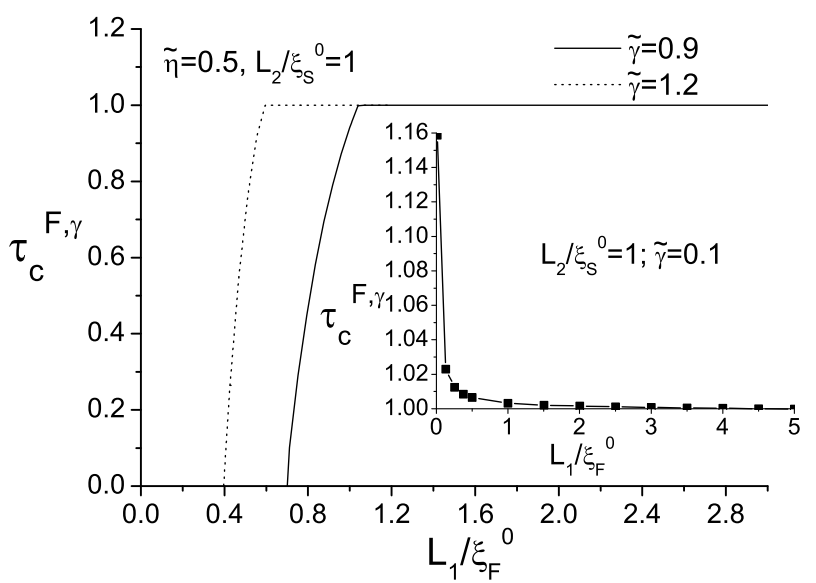

FIG. 7: Ferroelectric transition temperature vs the FE-layer thickness (for a S-film with $L_{2} / \xi_{S}^{0}=1.0$ ) for $\tilde{\eta}=0.5$ in the strong coupling regime. The inset shows $\tau_{c}^{F, \gamma}$ in the weakcoupling regime for $\tilde{\gamma}=0.1$.

$L_{1}$ shown in Fig. ?? $(\tilde{\gamma} \geq 0.9)$, is governed by a decrease of the domain phase-region as $L_{1}$ increases (see the plots in Fig. (5). First, for thin FE-films, the sys- 

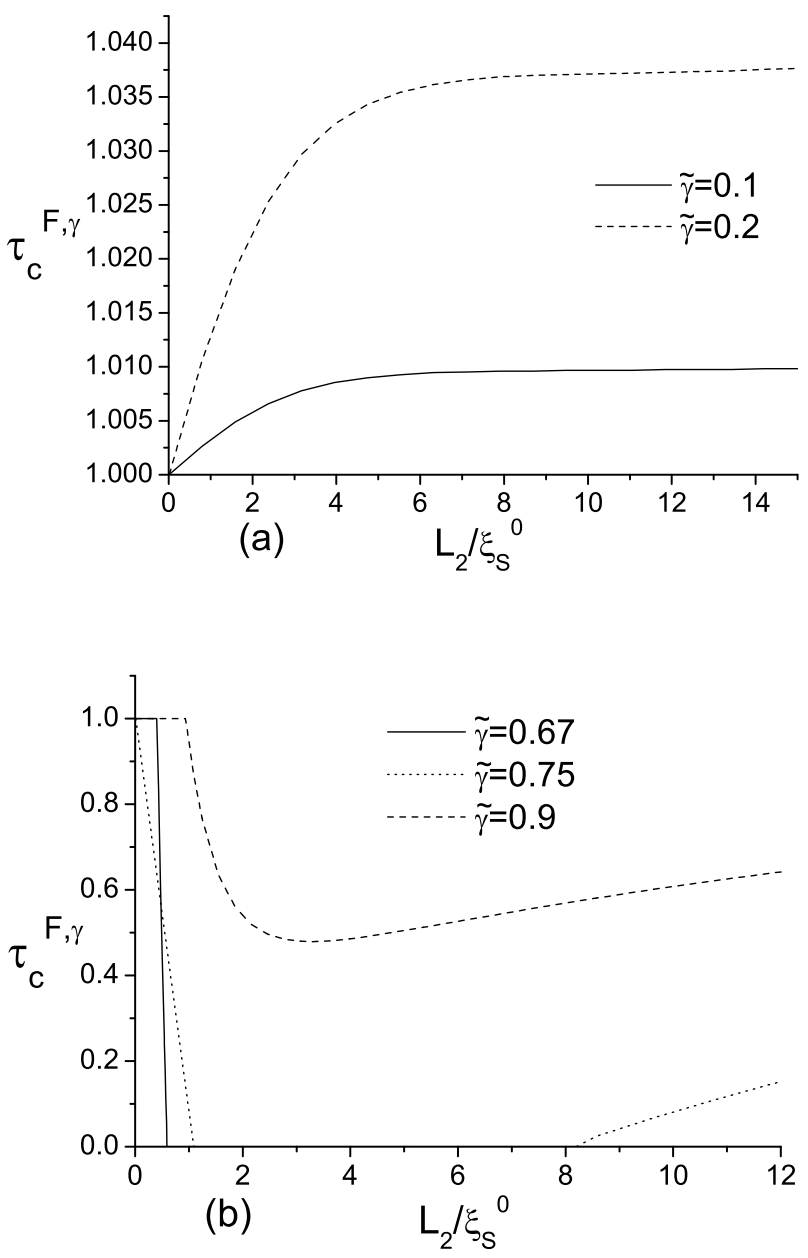

FIG. 8: Ferroelectric transition temperature vs the S-layer thickness (for a FE-film with $L_{1} / \xi_{F}^{0}=1.0$ ) for $\tilde{\eta}=0.5$ and different values of coupling strength $\tilde{\gamma}$ : (a) weak coupling regime and (b) strong coupling regime.

tem is in the domain phase already for $T \sim 0$. As $L_{1} / \xi_{F}^{0}$ increases, the area of the domain state shrinks towards higher $T$ and finally disappears, which results in an increase of $\tau_{c}^{F, \gamma}$ up to $\tau_{c}^{F}=1$ related to the transition from the $\mathrm{FE}+\mathrm{NM}$ (suppressed) to the paraelectric state (see the corresponding diagram in Fig. 5(c)). Note that for a small thickness of the FE-film $\left(L_{1} / \xi_{F}^{0} \sim\right.$ $0.5-1$ in Fig. ??), the behavior of $\tau_{c}^{F, \gamma}$ reflects a "crossover" between these two different ferroelectric transition types, namely, between the transition from the $\mathrm{FE}+\mathrm{NM}$ (suppressed) to the domain state for the thin FE-films, and the transition from the $\mathrm{FE}+\mathrm{NM}$ to the paraelectric phase for the thick FE-layers. This effect is also seen in the large $\tilde{\gamma}$-region of the diagrams plotted in

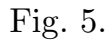

(4) Fig. ??(b) shows a nonmonotonic dependence of $\tau_{c}^{F, \gamma}$ on $L_{2}$ for $\tilde{\gamma}>\tilde{\gamma}_{c}$. As in the $L_{1}$-dependences, for a given value of $\tilde{\gamma}$ at a moderate thickness $L_{1} / \xi_{S}^{0} \approx 1$, we find similar, but reversed "crossover" between the two abovementioned different transition types. Specifically, while for thin S-films, the increase of $T$ results in the transition from the $\mathrm{FE}+\mathrm{NM}$ (suppressed) to the paraelectric state (at $\tau_{c}^{F, \gamma}=1$ ), the increase of the S-thickness induces the enhancement of the domain-type phase, leading to a lower $\tau_{c}^{F, \gamma}$ (which now corresponds to the transition between the $\mathrm{FE}+\mathrm{NM}$ and the domain phases). See the evolution of the phase diagram topology as $L_{2} / \xi_{S}^{0}$ varies, shown in Fig. [6] Furthermore, we note that for the larger $L_{2} / \xi_{S}^{0}>0.7$ the transition from the FE+NM- to the symmetric domain-state exhibits:

(i) monotonic decay of $\tau_{c}^{F, \gamma}$ and vanishing at $L_{2} / \xi_{S}^{0}=0.7$ (case $\tilde{\gamma}=0.67$ in Fig. ??(b));

(ii) reentrant behavior characterized by the vanishing of $\tau_{c}^{F, \gamma}$ in a certain interval $L_{2} / \xi_{S}^{0}\left(1<L_{2} / \xi_{S}^{0}<8.2\right.$ for $\tilde{\gamma}=0.75$ in Fig. ??(b)) and finite $\tau_{c}^{F, \gamma}$ otherwise;

(iii) for the larger values of $\tilde{\gamma}(\tilde{\gamma}=0.9$ in Fig. ??(b)), $\tau_{c}^{F, \gamma}$ first decays to a finite value exhibiting a minimum at a particular $L_{2}\left(L_{2} / \xi_{S}^{0} \approx 3\right)$, and then increases again with a further increase of the S-thickness, approaching a saturation value for thick S-layers. This behavior has a simple physical explanation: once the domain state is expanded down to $T=0$ (yielding $\tau_{c}^{F, \gamma}=0$ at $\tilde{\gamma}=0.75$ and $\left.L_{2} / \xi_{S}^{0}=1.0\right)$ the further increase of $L_{2}$ for strong coupling leads to the larger spatial inhomogeneous terms in the FE-energy (2), which results in a slight enhancement of the suppressed state towards higher $T$ and as a result, to an increase of $\tau_{c}^{F, \gamma}$ (see the two phase diagrams for $L_{2} / \xi_{S}^{0}=5$ and $L_{2} / \xi_{S}^{0}=15$ shown in Fig. [6(a)).

\section{CONCLUSIONS}

In this paper we have developed a phenomenological model describing periodic ferroelectric-superconductor multilayers. This model generalizes in some aspects the microscopic approach proposed in Ref. 24 where the superconducting layers are represented by two planes with a possibility of charge transfer between the planes and the interior of the layer. We analyze here the case when the ferroelectric transition temperature lies below the temperature of the transition to the superconducting state $\left(T_{c}^{F}<T_{c}^{S}\right)$ which is realized in some complex insulating perovskite alloys such as $\mathrm{Ba}_{x} \mathrm{Sr}_{1-x} \mathrm{TiO}_{3}$ (BST) coupled to high-temperature superconductors. Similarly to the microscopic model, we observe the stabilization of the symmetric domain-type phase exhibiting a weak polarization due to the FE-S contacts above the bulk ferroelectric transition temperature. On the other hand, we find the polarization-induced suppression of the superconductivity in the S-layer. We show that the ultimate reason for the destruction of the superconducting state for strong FE-S coupling, is the increase of the contribution of the spatial inhomogeneities to the energy of the system.

We study the behavior for different thicknesses of the $\mathrm{FE}$ and S-layer demonstrating the strong change of the 
topology of the phase diagrams $(\tilde{\gamma}, \tau)$ with a variation of $L_{1} / \xi_{F}^{0}$ and $L_{2} / \xi_{S}^{0}$.

In general, we find several types of $T_{c}^{F, \gamma}\left(L_{1}\right)$ and $T_{c}^{F, \gamma}\left(L_{2}\right)$ behavior. For the weak FE-S coupling, $\tilde{\gamma}<\tilde{\gamma}_{c}$, we obtain a monotonic variation of $T_{c}^{F, \gamma}$, with approaching saturation value in the system with the thick FE or $\mathrm{S}$ layer. In contrast to this, for the strong coupling, $\tilde{\gamma}>\tilde{\gamma}_{c}$, the dependence of $T_{c}^{F, \gamma}$ vs layer thicknesses appears to be highly nonmonotonic due to the competition between the first tendency to expand the domain state (prevailing for the thick S-layers) and the second tendency to destroy the superconductivity dominating for the thicker FE-layers.

\section{ACKNOWLEDGEMENTS}

This work has been supported by the Schwerpunktprogramm of the German Science Foundation (Deutsche Forschungsgemeinschaft) under Grant No. SPP-1056.

\section{APPENDIX: DETERMINATION OF PHASE $\phi$ IN THE CASE WITHOUT MAGNETIC FIELD}

Let us consider in more details the superconducting layer without a magnetic field. The general form of the Ginzburg-Landau functional in the S-layer can be written as

$$
\begin{aligned}
& F_{S}=\int_{L_{1}}^{L} d z\left[\frac{1}{2} a_{S}|\psi(z)|^{2}+\frac{1}{4} b_{S}|\psi(z)|^{4}\right. \\
& \left.+\frac{\hbar^{2}}{2 m e}\left|\left(\frac{d}{d z}-\frac{2 i e}{\hbar c} A(z)\right) \psi(z)\right|^{2}\right]
\end{aligned}
$$

where $\psi(z)=|\psi(z)| \exp (i \phi(z))$.
Variation of (??) with respect to $A, \psi$ and $\psi^{*}$ gives the following Ginzburg-Landau equations for the determination of $A,|\psi|$ and $\phi$ :

$$
\begin{aligned}
& \frac{2 e}{\hbar c} A(z)-\phi^{\prime}(z)=0, \\
& \left(a_{S}+b_{S}|\psi|^{2}\right)|\psi| \cos \phi-\frac{\hbar^{2}}{m e} \\
& \times\left(\psi^{\prime \prime} \cos \phi-\psi^{\prime} \phi^{\prime} \sin \phi-\left(\phi^{\prime}\right)^{2} \psi \cos \phi\right)=0, \\
& \left(a_{S}+b_{S}|\psi|^{2}\right)|\psi| \sin \phi-\frac{\hbar^{2}}{m e} \\
& \times\left(\psi^{\prime \prime} \sin \phi+\psi^{\prime} \phi^{\prime} \cos \phi-\left(\phi^{\prime}\right)^{2} \psi \sin \phi\right)=0 .
\end{aligned}
$$

Note that in the obtained in this case boundary conditions the terms containing $\phi$ and $\phi^{\prime}$ appear with the opposite signs and thus cancel, resulting in the standard form given by (21) and (22) in the paper.

From (??) we immediately obtain that the single possible solution for $\phi$ is:

$$
\phi=\phi_{0}=\text { const }, \quad L_{1}<z<L .
$$

We assume here for simplicity the absence of the tunneling current through the insulating FE-layer, which corresponds to the zero phase difference on the contacts between the S-films:

$$
\Delta \phi=\phi\left(L_{1}^{+}\right)-\phi\left(0^{-}\right)=0
$$

and leads to the constant phase in the whole system: $\phi \equiv \phi_{0}$. As the result, we get from (??)

$$
A(z)=\frac{\hbar c}{2 e} \phi^{\prime}(z)=0
$$

and the next two equations transform into the equation (20) for the absolute value of the superconducting order parameter $|\psi(z)|$.
* Electronic address: natalie@ph.tum.de

1 O.G. Vendik, E.K. Hollmann, A.B. Kozyrev, and A.M. Prudan, J. Superconduct. 12, 325 (1999).

2 O.G. Vendik, L.T. Ter-Martirosyan, A.I. Dedyk, S.F. Karmanenko, and R.A. Chakalov, Ferroelectrics 144, 337 (1993).

3 P.Kr. Petrov, E.F. Carlsson, P. Larsson, M. Friesel, and Z.G. Ivanov, J. Appl. Phys. 84, 3134 (1998).

${ }^{4}$ L. Ryen, E. Olsson, C.N.L. Edvardsson, and U. Helmersson, Physica C 304, 307 (1998).

5 P.K. Petrov, Z.G. Ivanov, and S.S. Gevorgian, Materials Sci. Eng. A 288, 231 (2000).

${ }^{6}$ F.W. Van Keuls, R.R. Romanofsky, D.Y. Bohman, M.D. Winters, F.A. Miranda, C.H. Mueller, R.E. Treece, T.V. Rivkin, and D. Galt, Appl. Phys. Lett. 71, 3075 (1997).

7 P. Konsin and B. Sorkin, Phys. Rev. B 58, 5795 (1998).

8 P. Konsin and T. Örd, Physica C 191, 469 (1992).

9 W.G. Jenks and L.R. Testardi, Phys. Rev. B 48, 12993
(1993)

10 J. Mannhart, Supercond. Sci. Technol. 9, 49 (1996).

11 G.L. Larkins, M.Y. Avello, J.B. Boyce, and D.K. Fork, IEEE Trans. Appl. Supercond. AS-5, 3049 (1995).

12 V.V. Lemanov and A.L. Kholkin, Phys. Solid State 36, 841 (1994).

13 V.V. Lemanov, A.L. Kholkin, and A.B. Sharman, JETP Lett. 56, 562 (1992).

14 X.X. Xi, C. Doughty, A. Walkenhorst, C. Kwon, Q. Li, and T. Venkatesan, Phys. Rev. Lett. 68, 1240 (1992).

15 K. Nakajima, K. Yokota, H. Myoren, J. Chen, and T. Yamashita, Appl. Phys. Lett. 63, 684 (1993).

16 J. Mannhart, J. Strobel, J.G. Bednorz, and Ch. Gerber, Appl. Phys. Lett. 62, 630 (1993).

17 Y. Watanabe, Appl. Phys. Lett. 66, 1770 (1995).

18 D. Matthey, S. Gariglio, C.H. Ahn, and J.-M. Triscone, Physica C 372-376, 583 (2002).

19 Y. Watanabe, Y. Matsumoto, M. Tanamura, H. Asami, and A. Kato, Physica C 235-240, 739 (1994). 
20 V. Muller, C. Hucho, and D. Maurer, Ferroelectrics 130, 45 (1992).

21 A. Bussmann-Holder, A. Simon, and H. Buttner, Phys. Rev. B 39, 207 (1989).

22 G.C. Vezzoli, F. Craver, M.F. Chen, and W. Stanley, Ferroelectrics 130, 215 (1992).

23 O.G. Vendik and L.T. Ter-Martirosyan, Phys. Solid State 36, 1778 (1994).

24 N. Pavlenko and F. Schwabl, Phys. Rev. B 67, 094516 (2003).

25 L.D. Landau and E.M. Lifshitz, Electrodynamics of Continuous Media, (Pergamon Press, Oxford, 1960).

26 S.N. Artemenko and A.F. Volkov, Sov. Phys. Usp. 22, 295 (1979).
27 J.D. Jackson, Classical Electrodynamics, (Wiley \& Sons, New York, 1975).

${ }^{28}$ K. Kretschmer and K. Binder, Phys. Rev. B 20, 1065 (1979).

29 M. Tinkham, Introduction to Superconductivity, (McGrawHill, New York, 1996).

30 M.E. Lines and A.M. Glass, Principles and Applications of Ferroelectrics and Related Materials, (Clarendon Press, Oxford, 1977).

31 A. Gajevskis, I. Popova, M. Kalnberga, E. Birks, Z. Zaula, V. Zauls, G. Liberts, A. Sternberg, C. Czurda, E. Seidl, and H. Weber, Ferroelectrics 131, 375 (1992). 